\title{
Twisted mass QCD in the charm sector
}

\author{
Benoît Blossier* \\ DESY, Platanenallee 6, 15738 Zeuthen, Germany \\ E-mail: Benoit.Blossier@desy.de
}

\section{Gregorio Herdoiza}

INFN - Sezione di Roma Tor Vergata, Via della Ricerca Scientifica 1, I-00133 Roma, Italy

\section{Silvano Simula}

INFN - Sezione di Roma Tre, Via della Vasca Navale 84, I-00146 Roma, Italy

\section{for the European Twisted Mass Collaboration (ETMC)}

\begin{abstract}
We present preliminary results for the charm quark mass $m_{c}$ and the $D$ and $D_{s}$ mesons decay constants $f_{D}$ and $f_{D_{s}}$ from a lattice QCD calculation with $\mathrm{N}_{\mathrm{f}}=2$ dynamical fermions. We use the twisted mass fermionic action defined at maximal twist so that physical quantities are automatically $\mathscr{O}(a)$ improved. Two lattice spacings are considered. The charm quark mass has been renormalised in the RI-MOM scheme. After a matching to the $\overline{\mathrm{MS}}$ scheme, we obtain from the simulation at a fine lattice $(a \sim 0.09 \mathrm{fm}) m_{c}^{\overline{\mathrm{MS}}}\left(m_{c}\right)=1.481 \pm 0.022 \pm 0.092$ $\mathrm{GeV}, f_{D}=205 \pm 13 \pm 17 \mathrm{MeV}, f_{D_{s}}=271 \pm 6 \pm 6 \mathrm{MeV}$ and from the simulation at the finer lattice $(a \sim 0.07 \mathrm{fm}) m_{c}^{\overline{\mathrm{MS}}}\left(m_{c}\right)=1.474 \pm 0.041 \pm 0.132 \mathrm{GeV}, f_{D}=230 \pm 31 \pm 8 \mathrm{MeV}$ and $f_{D_{s}}=264 \pm 5 \pm 8 \mathrm{MeV}$. We chose three renormalisation conditions to determine $m_{c}$ : the spread between the final results contributes to the systematic error. At both lattice spacings, particularly at the finer one, the error on $m_{c}$ is dominated by present uncertainty on the renormalisation constant $Z_{P}$, which should be reduced before performing a reliable continuum limit.
\end{abstract}

XXV International Symposium on Lattice Field Theory

30 July - 4 August 2007

University of Regensburg, Germany

\footnotetext{
* Speaker.
} 


\section{Introduction}

The physics of charm bound states regained recently the attention of particle physicists with the discovery of the new resonances $X(3872), X(3943), Y(3940), Y(4260)$ and $Z(3930)$ [1] and of a very narrow scalar state $D_{s}(2317)$ [沟], whose composition is still an open question. The experimental evidence for oscillations in the $D^{0}-\overline{D^{0}}$ system [3] might be the fir rst signal for physics beyond the Standard Model in the charm sector. Even if it is expected that the long-distance physics is a dominant effect in that process, the $\Delta C=2$ contribution to $x_{D}=\Delta M_{D} / \Gamma_{D}$ might be not negligible. It is given by a box diagram, as in the $B-\bar{B}$ system, and it is proportional to $f_{D}^{2}$. In the CKM matrix, $V_{c s}$ is one of the elements having the largest uncertainty when one does not impose the $3 \times 3$ unitarity: $\frac{\Delta\left|V_{c s}\right|}{\left|V_{c s}\right|}=9.82 \%$ [ $\llbracket$ ]: most of it comes from the theory. An appropriate way to extract $V_{c s}$ is to measure the leptonic decay width $D_{s} \rightarrow l \bar{v}$, which however requires the estimation of the decay constant $f_{D_{s}}$. Eventually it is well established that $V_{c b}$ can be constrained by analysing the inclusive semileptonic decay $\bar{B} \rightarrow X_{c} l \bar{v}$. An OPE is used in the Heavy Quark Expansion (HQE) and the total branching ratio reads $\left[\right.$ ॠ $\mathrm{BR}_{\mathrm{SL}}(\bar{B})=\mathrm{BR}_{\mathrm{SL}}^{(0)}[1+f(r)], r=\left(\frac{m_{c}}{m_{b}}\right)^{2}$, where $\mathrm{BR}_{\mathrm{SL}}^{(0)}$ is the dominant term in the $m_{c} / m_{b}$ expansion.

From those phenomenological aspects we conclude that it is important to determine as precisely as possible the decay constants $f_{D}, f_{D_{s}}$ and the charm quark mass $m_{c}$. That quark is of course too heavy to make predictions by using Chiral Perturbation Theory $(\chi \mathrm{PT})$ and too light to compute the amplitudes by using only the HQE: corrections of $\mathscr{O}\left(\Lambda_{Q C D} / m_{c}\right)^{n}$ and $\mathscr{O}\left(m_{c} / m_{b}\right)^{n}$ might be larger than the precision of few \% that we want to reach.

Lattice QCD is a good tool to study the charm sector. However the continuum limit can be diffi cult to reach because of large cut off effects (typically, $0.2<a m_{c}^{0}<0.4$ ). It is therefore crucial to improve the action and the currents regularised on the lattice. Several theories proposed in the literature so far have the common property that they require the tuning of a certain number of parameters to achieve improvement, by applying the Symanzik's program [6]. On the other side it has been shown [7] that Twisted mass QCD (TmQCD) [8, 9] requires the tuning of a single parameter (the untwisted mass $m_{0}$ ), so that hadronic quantities like the pseudoscalar meson masses and decay constants are automatically $\mathscr{O}(a)$ improved at maximal twist. Other nice properties of such an action are that the physical quark mass is related to the twisted mass parameter of the action, its renormalisation is only multiplicative and the pseudoscalar decay constant does not require the introduction of any renormalisation constant [9]: for two quark flavors 1 and 2 (for example a light flavor $\ell$ and a heavy flavor $h$ ) it is simply given by

$$
f_{P S}\left(\mu_{1}, \mu_{2}\right)=\frac{\mu_{1}+\mu_{2}}{m_{P S}^{2}\left(\mu_{1}, \mu_{2}\right)}\left|\left\langle 0\left|P^{c}(0)\right| P\right\rangle\right|, \quad P^{c}=\bar{\psi}_{1}(r) \gamma^{5} \psi_{2}(-r),
$$

where $r$ is the Wilson parameter and we defi ne the composite operator $P$ in the physical basis.

We present a preliminary lattice QCD determination of the charm quark mass $m_{c}$ and the decay constants $f_{D}$ and $f_{D_{s}}$. We have performed full dynamical simulations for $\mathrm{N}_{\mathrm{f}}=2$ light degenerate sea quarks; the strange and the charm quarks have been added in the valence sector. The calculation is based on the analysis of the gauge confi gurations ensembles $B_{1}-B_{5}$ and $C_{1}-C_{4}$ [10] (240 and 130 confi gurations of $B_{1}-B_{5}$ and $C_{1}-C_{4}$, respectively, have been analysed) which have been generated with the TISym gauge action at $\beta=3.9(a=0.0855(5)(31) \mathrm{fm})$ and $\beta=4.05(a=$ 


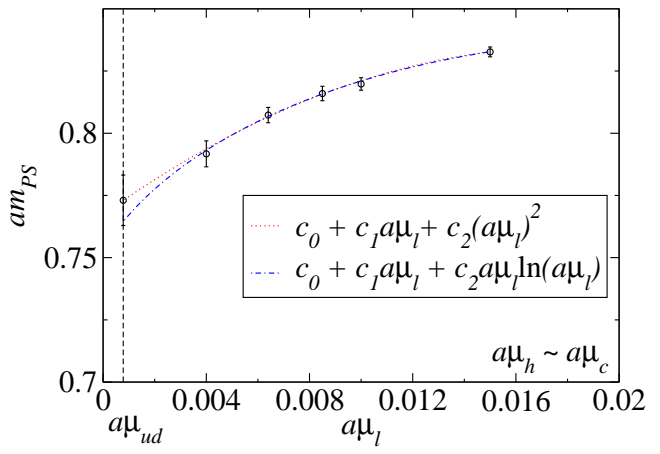

(a)

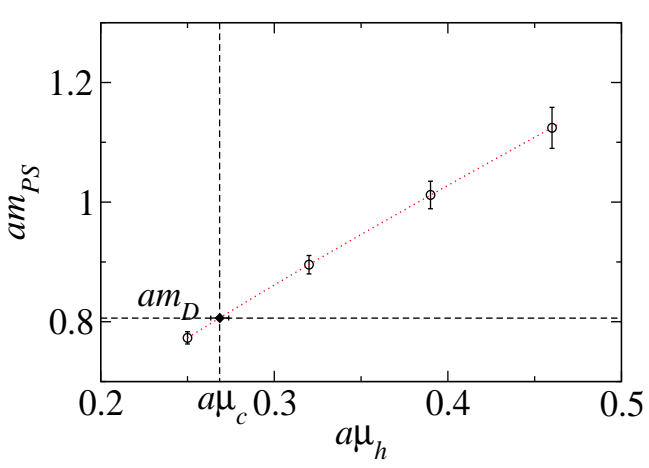

(b)

Figure 1: (a) Chiral extrapolation of the pseudoscalar heavy-light meson masses $(\beta=3.9)$. (b) Pseudoscalar heavy-light meson mass in function of the heavy quark mass $(\beta=3.9)$.

$0.0667(5)(24) \mathrm{fm})$ respectively and the twisted mass fermionic action defi ned at maximal twist. The light quark masses are in the range $\left[m_{s} / 6,2 m_{s} / 3\right]$, to perform the chiral extrapolation, and we used masses around the strange mass and the charm mass to do the appropriate interpolations.

At each sea quark mass we have computed the two-point correlation functions of pseudoscalar mesons. Each measurement has been separated by $20 \mathrm{HMC}$ trajectories, which is enough to avoid autocorrelation time effects. The statistical accuracy has been improved by using all to all stochastic propagators. Statistical errors on the meson masses and decay constants are evaluated at a given sea quark mass by using a jacknife procedure with 10 measurements discarded in each bin. The error obtained after a combination of data coming from simulations with different sea quark masses (i.e. statistically independent) is computed using a bootstrap method.

\section{Charm quark mass}

To estimate the charm quark mass we use the following strategy: we compute the pseudoscalar meson mass $m_{P S}\left(\mu_{\text {sea }}, \mu_{\ell}, \mu_{h}\right)$ (where $\ell$ and $h$ are valence light and heavy quarks respectively) at the points $\mu_{\ell}=\mu_{\text {sea }}$, which allows us to extrapolate down to the physical light quark mass $\mu_{u d} \equiv \frac{\mu_{u}+\mu_{d}}{2}$, previously determined in [11]. Once the dependence on the light quark mass has been taken into account, one studies the dependence of $m_{P S}$ on the heavy mass $\mu_{h}$. The bare charm quark mass $\mu_{c}$ is determined by using the following condition: $m_{P S}\left(\mu_{u d}, \mu_{c}\right)=m_{D}$.

We have performed a quadratic extrapolation of $m_{P S}\left(\mu_{\ell}=\mu_{\text {sea }}, \mu_{h}\right)$ in $\mu_{\ell}$ down to $\mu_{\ell}=\mu_{u d}$. This is illustrated in Figure 1 (left). We have also introduced a logarithmic dependence on $\mu_{\ell}$ : $m_{P S}\left(\mu_{\ell}, \mu_{h}\right)=c_{0}\left(\mu_{h}\right)+a \mu_{\ell}\left[c_{1}\left(\mu_{h}\right)+c_{2}\left(\mu_{h}\right) \ln \left(a \mu_{\ell}\right)\right]$. As a third possibility we have done a simple linear extrapolation. The spread between these different fi ts is included in the systematic error at the end of the computation. We then performed a fit of $m_{P S}$ as a function of $\mu_{h}$ : we used either a quadratic polynomial in $\mu_{h}$, or a quadratic polynomial in $\frac{1}{\mu_{h}}$, or also, as a third ansatz, $m_{P S}=d_{0}+d_{1} \mu_{h}+\frac{d_{2}}{\mu_{h}}$. We show in Figure 1 (right) the quality of the latter fi t function, that appears to be the most appropriate to describe the data. Once the bare charm quark mass $\mu_{c}$ is extracted, we renormalise it in the RI-MOM scheme: $m_{c}^{\mathrm{RI}-\mathrm{MOM}}=Z_{\mu}^{\mathrm{RI}-\mathrm{MOM}} \mu_{c}$ where $Z_{\mu}^{\mathrm{RI}-\mathrm{MOM}}=1 / Z_{P}^{\mathrm{RI}-\mathrm{MOM}}$ in TmQCD [7]. Finally we perform a matching onto the $\overline{\mathrm{MS}}$ scheme. We indicate in Table 1 the value of $m_{c}^{\overline{\mathrm{MS}}}\left(m_{c}\right)$ for the two lattice spacings, knowing that $Z_{P}^{\mathrm{RI}-\mathrm{MOM}}(\beta=3.9,1 / a)=0.39(1)(2)$ 


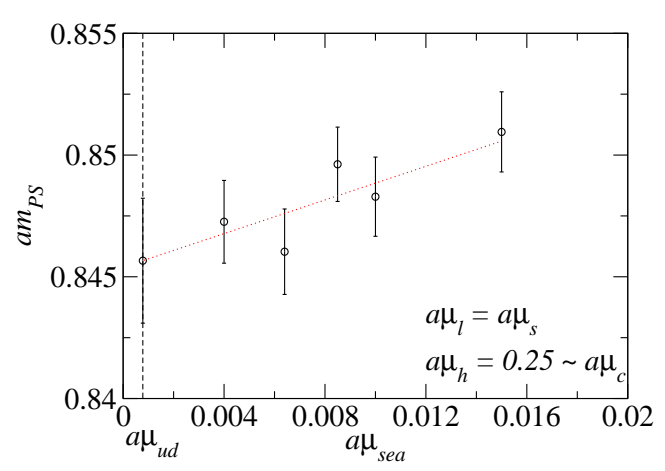

(a)

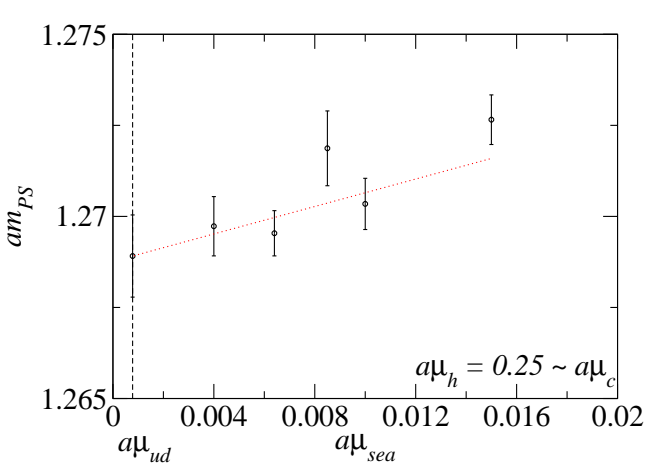

(b)

Figure 2: (a) Chiral extrapolation of $m_{P S}\left(\mu_{\text {sea }}, \mu_{\ell}=m_{s}, \mu_{h} \sim m_{c}\right)(\beta=3.9)$. (b) Chiral extrapolation of $m_{P S}\left(\mu_{\text {sea }}, \mu_{h}, \mu_{h}\right)(\beta=3.9)$.

and the preliminary value of $Z_{P}^{\mathrm{RI}-\mathrm{MOM}}(\beta=4.05,1 / a)=0.40(1)(4)^{1}\left[12\right.$. The fi rst error on $m_{\epsilon}$ is statistical, the second is the systematic error coming from $Z_{P}$, the third comes from the uncertainty on $a$ and the last one is the systematic error from the chiral extrapolation. In that table we have also collected the result of two other determinations of $m_{c}$, by using the following renormalisation conditions:

1) $m_{P S}\left(\mu_{\text {sea }}=\mu_{u d}, \mu_{\ell}=\mu_{s}, \mu_{c}\right)=m_{D_{s}}$ and 2) $m_{P S}\left(\mu_{\text {sea }}=\mu_{u d}, \mu_{c}, \mu_{c}\right)=m_{\eta_{c}}$.

$\mu_{s}$ is the bare strange quark mass which has been determined in [14]. The heavy-heavy pseudoscalar meson correlator has been computed by using the interpolating fi eld $\bar{\psi}_{h}(r) \gamma^{5} \psi_{h}(r)$.

The dependence of those two observables on the sea quark mass is very weak, as shown in Figure 2 . We used a linear fi t in $\mu_{\ell}$ and $\mu_{\text {sea }}$ to interpolate to $\mu_{s}$ and to extrapolate down to $\mu_{u d}$ respectively:

$$
\begin{aligned}
& m_{P S}\left(\mu_{\text {sea }}, \mu_{\ell}, \mu_{h}\right)=p_{0}\left(\mu_{h}\right)+a \mu_{\ell} p_{1}\left(\mu_{h}\right)+a \mu_{\text {sea }}\left[p_{2}\left(\mu_{h}\right)+a \mu_{\ell} p_{3}\left(\mu_{h}\right)\right], \\
& m_{P S}\left(\mu_{\text {sea }}, \mu_{h}, \mu_{h}\right)=q_{0}\left(\mu_{h}\right)+a \mu_{\text {sea }} q_{1}\left(\mu_{h}\right) .
\end{aligned}
$$

The uncertainty coming from the chiral extrapolation is quite reduced compared to $m_{P S}\left(\mu_{\text {sea }}=\right.$ $\left.\mu_{\ell}, \mu_{h}\right)$. However we introduce a small uncertainty from $\mu_{s}$ on the fi rst observable (third error on $\left.m_{c}^{\overline{\mathrm{MS}}}\left(m_{c}, m_{D_{s}}\right)\right)$ and from the disconnected diagram which contributes to the second but that we did not compute. The last error on $m_{c}^{\overline{\mathrm{MS}}}\left(m_{c}, m_{D_{s}}\right)$ and $m_{c}^{\overline{\mathrm{MS}}}\left(m_{c}, m_{\eta_{c}}\right)$ comes from the uncertainty on $a$. We have not performed a continuum limit extrapolation yet because only 2 lattice spacings have been considered so far and the uncertainty on $Z_{P}$ is still rather large, especially at $\beta=4.05$. Concerning cut off effects, it is remarkable that they appear to be rather weak on the unrenormalised charm mass at $\beta=4.05$ : indeed, the value of $\mu_{c}$ extracted from the 3 observables are much closer at this $\beta$ than at $\beta=3.9$.

To conclude this section we note that our values of $m_{c}$ are large with respect to most of the recent lattice estimations [15]: however the currently large uncertainty on $Z_{P}$ at $\beta=4.05$ implies that any conclusion about the continuum limit result would be untimely.

\footnotetext{
${ }^{1}$ At this $\beta$ a chiral extrapolation has been performed in the valence sector, at $a \mu_{\text {sea }}=0.003$, but not yet in the sea sector. However it was found at $\beta=3.9$ that $Z_{P}$ depends only weakly on the sea quark mass. Thus as a first step of the analysis we will include the sea effects in the systematic uncertainty. Moreover, an alternative estimate of $Z_{P}$ at $\beta=4.05$ may come from scaling as described in [13], which brings our fi nal estimate of the systematic error to 0.04 .
} 

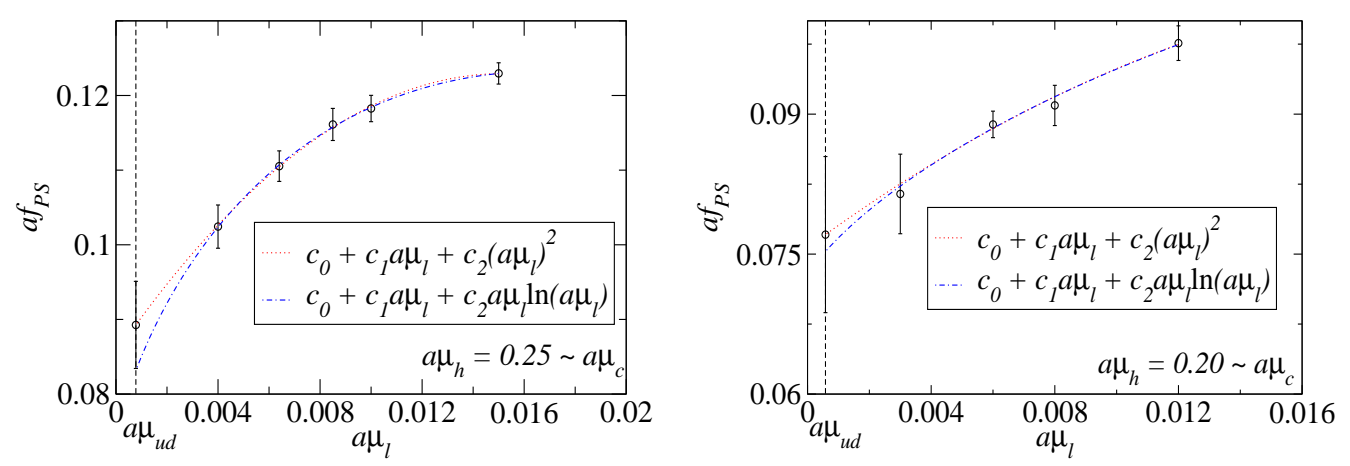

Figure 3: Chiral extrapolation of $f_{P S}\left(\mu_{\ell}=\mu_{\text {sea }}, \mu_{h} \sim \mu_{c}\right)$ at $\beta=3.9$ (left) and $\beta=4.05$ (right).

\section{Heavy-light meson decay constants}

To determine $f_{D}$ and $f_{D_{s}}$ we employ the same strategy as in the previous section, using eq. (1.1). We show in Figure 3 the chiral extrapolation of $f_{P S}\left(\mu_{\text {sea }}=\mu_{\ell}, \mu_{h}\right)$ at $\mu_{h} \sim \mu_{c}$ down to the physical light quark mass. We found that introducing a quadratic term in the extrapolation improves the fi t, particularly for the coarse lattice. As before, we also introduced also a logarithmic dependence on $\mu_{\ell}$ in the fit. We include the spread between the different chiral extrapolations in the systematic error.

At $\mu_{\ell} \sim \mu_{s}, f_{P S}\left(\mu_{\text {sea }}, \mu_{\ell}, \mu_{h}\right)$ has a similar linear dependence on $\mu_{\ell}$ to the one of $m_{P S}\left(\mu_{\text {sea }}, \mu_{\ell}, \mu_{h}\right)$. Moreover for both the dependence on $\mu_{\text {sea }}$ is weak and very well described by a linear fi $t$ as well. We performed a fi t of $f_{P S} \sqrt{m_{P S}}$ with a quadratic polynomial in $\frac{1}{m_{P S}}$ (we can not isolate any logarithmic dependence on $\alpha_{S}\left(m_{P S}\right)$ in our range of heavy masses): $f_{P S} \sqrt{m_{P S}}=f_{0}+\frac{f_{1}}{m_{P S}}+\frac{f_{2}}{m_{P S}^{2}}$. We show in Figure 4 the quality of the fit for $f_{P S} \sqrt{m_{P S}}\left(\mu_{u d}, \mu_{h}\right)$.

We give in Table 2 our values of $f_{D}, f_{D_{s}}$ and $f_{D_{s}} / f_{D}$. The fir rst error is a statistical error, the second error on $f_{D_{s}}$ and on $f_{D_{s}} / f_{D}$ comes from the uncertainty on the bare strange quark mass, the second error on $f_{D}$ and the third error on $f_{D_{s}}$ come from the uncertainty on the lattice spacing. The last error on $f_{D}$ and $f_{D_{s}} / f_{D}$ comes from the spread between different chiral fi ts. We have collected the most recent lattice estimations of those quantities in Figure 5 [16]. On the experimental side

\begin{tabular}{|c|c|c|c|}
\hline$\beta$ & $m_{c}^{\overline{\mathrm{MS}}}\left(m_{c}, m_{D}\right)$ & $m_{c}^{\overline{\mathrm{MS}}}\left(m_{c}, m_{D_{s}}\right)$ & $m_{c}^{\overline{\mathrm{MS}}}\left(m_{c}, m_{\eta_{c}}\right)$ \\
\hline 3.9 & $1.481(22)(63)(8)(27) \mathrm{GeV}$ & $1.450(12)(61)(10)(15) \mathrm{GeV}$ & $1.420(5)(60)(6) \mathrm{GeV}$ \\
4.05 & $1.474(41)(129)(15)(5) \mathrm{GeV}$ & $1.498(6)(130)(12)(18) \mathrm{GeV}$ & $1.479(2)(129)(8) \mathrm{GeV}$ \\
\hline
\end{tabular}

Table 1: Charm quark mass fixed by using different observables.

\begin{tabular}{|c|c|c|}
\hline$\beta$ & 3.9 & 4.05 \\
\hline$f_{D}$ & $205(13)(3)(17) \mathrm{MeV}$ & $230(31)(6)(6) \mathrm{MeV}$ \\
$f_{D_{s}}$ & $271(6)(4)(5) \mathrm{MeV}$ & $264(5)(4)(7)$ \\
$\frac{f_{D_{s}}}{f_{D}}$ & $1.35(4)(1)(7)$ & $1.13(28)(2)(2)$ \\
\hline
\end{tabular}

Table 2: Decay constants $f_{D}$ and $f_{D_{s}}$ and $f_{D_{s}} / f_{D}$ from our simulation 


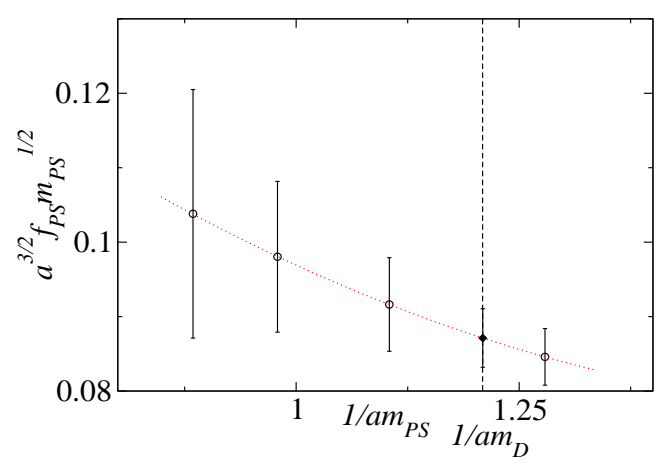

Figure 4: Scaling law of $f_{P S} \sqrt{m_{P S}}\left(\mu_{u d}, \mu_{h}\right)$ as a function of $1 / m_{P S}\left(\mu_{u d}, \mu_{h}\right)$ at $\beta=3.9$.

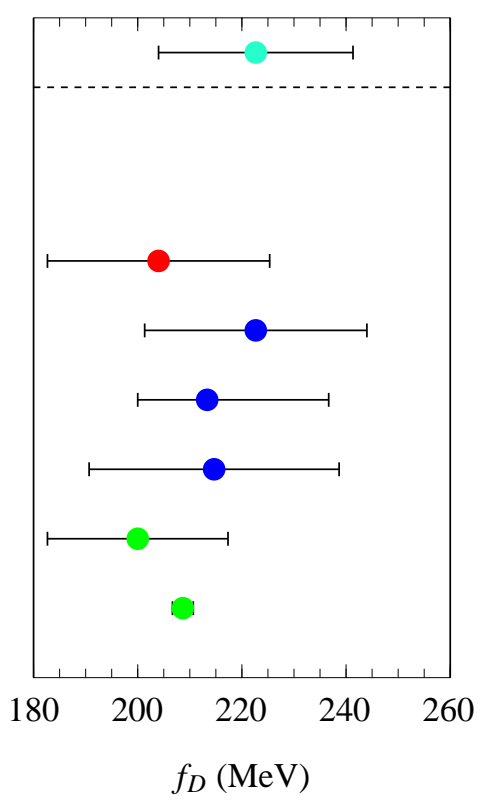

CLEO-c, 2005

Alpha, $\mathrm{N}_{\mathrm{f}}=0,2003$

Rome 2, $\mathrm{N}_{\mathrm{f}}=0,2003$

QCDSF, $\mathrm{N}_{\mathrm{f}}=0,2007$

CP-PACS, $\mathrm{N}_{\mathrm{f}}=2,2000$

MILC, $\mathrm{N}_{\mathrm{f}}=2,2002$

ETMC, $\mathrm{N}_{\mathrm{f}}=2,2007$

FNAL/MILC, $\mathrm{N}_{\mathrm{f}}=2+1,2005$

$\mathrm{HPQCD} / \mathrm{UKQCD}, \mathrm{N}_{\mathrm{f}}=2+1,2007$

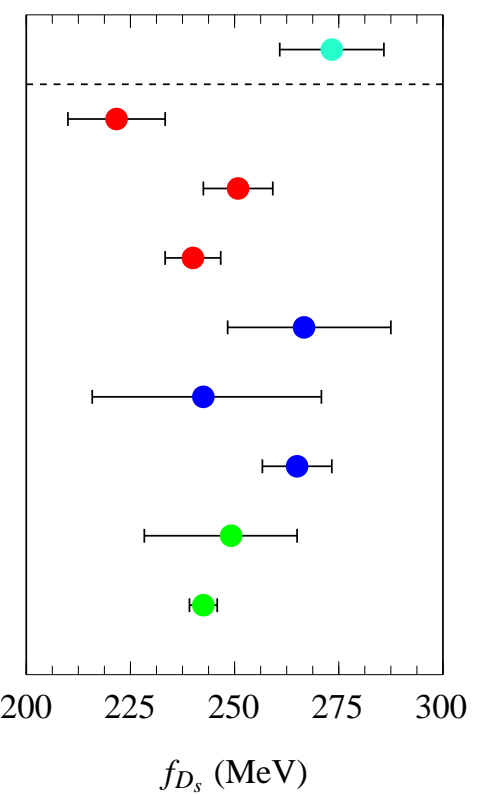

Figure 5: Recent lattice computations and experimental measurements of the decay constants $f_{D}$ (left panel) and $f_{D_{s}}$ (right panel). The ETMC values that we indicate are obtained by doing an average of the data at $\beta=3.9$ and $\beta=4.05$.

CLEO-c measured $f_{D}=222.6 \pm 16.7_{-3.4}^{+2.8} \mathrm{MeV}$ [17], $f_{D_{s}}=274 \pm 13 \pm 7 \mathrm{MeV}$ [18], which is a combination of analysis of $D_{s} \rightarrow \mu$ and $D_{s} \rightarrow \tau$ leptonic decays. Note that BABAR measured $f_{D_{s}}=283 \pm 17 \pm 7 \pm 14 \mathrm{MeV}[19]$.

\section{Summary}

We have presented preliminary results of a lattice computation of the charm quark mass $m_{c}$ and the $D$ and $D_{s}$ mesons decay constants $f_{D}$ and $f_{D_{s}}$ by using the $\mathrm{N}_{\mathrm{f}}=2$ TmQCD action defin ned at maximal twist. Encouraging results are found concerning cut off effects. Indeed, the bare charm quark mass extracted from 3 different observables looks consistent at the fi ner lattice. However, before performing the continuum limit on $m_{c}, f_{D}$ and $f_{D_{s}}$, we still have to reduce as much as possible the uncertainty on the renormalisation constant $Z_{P}$ and to increase the statistics at $\beta=4.05$. A more detailed study of the light quark dependence of the $D$ mesons masses and decay constants, 
based on heavy-light chiral perturbation theory, is still missing at this stage. Finally a better control on the continuum limit extrapolation will come from the on-going analysis of the data at a coarser lattice $(a \sim 0.1 \mathrm{fm})$.

\section{References}

[1] S. K. Choi et al (Belle Collaboration), Phys. Rev. Lett. 91, 262001 (2003); S. K. Choi et al (Belle Collaboration), Phys. Rev. Lett. 94, 182002 (2005); B. Aubert et al (BABAR Collaboration), Phys. Rev. Lett. 95, 142001 (2005); K. Abe et al (Belle Collaboration), [hep-ex/0507019]; S. Uehara et al (Belle Collaboration), Phys. Rev. Lett. 96, 082003 (2006).

[2] B. Aubert et al (BABAR Collaboration), Phys. Rev. Lett. 90, 242001 (2003).

[3] M. Staric et al (Belle Collaboration), Phys. Rev. Lett. 98, 211803 (2007); B. Aubert et al (BABAR Collaboration), [hep-ex/0703020].

[4] W. M. Yao et al, Journal of Physics G 33, 1 (2006).

[5] I. I. Bigi, M. Shifman, N. G. Uraltsev and A. Vainshtein, Phys. Rev. Lett. 71, 496 (1993).

[6] K. Symanzik, Nucl. Phys. B 226, 187 (1983); Nucl. Phys. B 227, 205 (1983).

[7] R. Frezzotti and G. C. Rossi, JHEP 0408, 007 (2004) [arXiv:hep-lat/0306014].

[8] S. Aoki, Phys. Rev. D 30, 2653 (1984).

[9] R. Frezzotti, P. A. Grassi, S. Sint and P. Weisz [Alpha Collaboration], JHEP 0108, 058 (2001) [arXiv:hep-lat/0101001].

[10] C. Urbach, PoS (LATTICE 2007)022.

[11] Ph. Boucaud et al, [ETM Collaboration], Phys. Lett. B 650, 304 (2007) [arXiv:hep-lat/0701012].

[12] P. Dimopoulos et al, PoS (LATTICE 2007)241.

[13] P. Dimopoulos et al, PoS (LATTICE 2007)102.

[14] B. Blossier et al [ETM Collaboration], [arXiv:0709.4574]; V. Lubicz et al, PoS (LATTICE 2007)374.

[15] D. Becirevic, V. Lubicz and G. Martinelli, Phys. Lett. B 524, 115 (2002) [arXiv:hep-ph/0107124]; J. Rolf and S. Sint [ALPHA Collaboration], JHEP 0212, 007 (2002) [arXiv:hep-ph/0209255]; G. M. de Divitiis, M. Guagnelli, R. Petronzio, N. Tantalo and F. Palombi, Nucl. Phys. B 675, 309 (2003) [arXiv:hep-lat/0305018]; A. Dougall, C. M. Maynard and C. McNeile, JHEP 0601, 171 (2006) [arXiv:hep-lat/0508033].

[16] A. Ali Khan et al., Phys. Lett. B 652, 150 (2007) [hep-lat/0701015]; G. M. de Divitiis, M. Guagnelli, F. Palombi, R. Petronzio and N. Tantalo, Nucl. Phys. B 672, 372 (2003) [arXiv:hep-lat/0307005]; A. Juttner and J. Rolf [ALPHA Collaboration], Phys. Lett. B 560, 59 (2003) [arXiv:hep-lat/0302016]; A. Ali Khan et al. [CP-PACS Collaboration], Phys. Rev. D 64, 034505 (2001) [arXiv:hep-lat/0010009]; C. Bernard et al. [MILC Collaboration], Phys. Rev. D 66, 094501 (2002) [arXiv:hep-lat/0206016]; C. Aubin et al., Phys. Rev. Lett. 95, 122002 (2005) [arXiv:hep-lat/0506030]; E. Follana et al., [arXiv:0706.1726].

[17] M. Artuso et al. [CLEO Collaboration], Phys. Rev. Lett. 95, 251801 (2005) [arXiv:hep-ex/0508057].

[18] T.K. Pedlar et al., [arXiv:0704.0439]; M. Artuso et al., [arXiv:0704.0629].

[19] B. Aubert et al. [BABAR Collaboration], Phys. Rev. Lett. 98, 141801 (2007) [arXiv:hep-ex/0607094]. 